\title{
Current Status and Recent Results of the COBRA Experiment
}

\author{
Daniel Muenstermann* on behalf of the COBRA collaboration ${ }^{\dagger}$ \\ Lehrstuhl fuer Experimentelle Physik IV, University of Dortmund, 44221 Dortmund, Germany \\ E-mail: Daniel.Muenstermann@physik.uni-dortmund.de
}

\begin{abstract}
The COBRA experiment is aiming to use a large amount of CdZnTe semiconductor detectors to perform a search for various double beta decay modes. The current status of the experiment and first results are presented. Improved world best half-life limits for the ground state transitions of ${ }^{64} \mathrm{Zn}$ and ${ }^{120} \mathrm{Te}$ for the $0 v \beta^{+} / E C$ decay channel and a measurement of the ${ }^{113} \mathrm{Cd}$ decay have been obtained. A brief outlook on future activities is presented.
\end{abstract}

International Europhysics Conference on High Energy Physics

July 21st - 27th 2005

Lisboa, Portugal

* Speaker.

$\dagger$ COBRA collaboration: J. Dawson, C. Montag, D. Palzeaird, C. Reeve, J. Wilson, K. Zuber (University of Sussex, UK), C. Goessling, H. Kiel, D. Muenstermann, S. Oehl, T. Villett (University of Dortmund, Germany), P. Harrison, B. Morgan, D. Stewart, Y. Ramachers (University of Warwick, UK), T. Bloxham, M. Freer (University of Birmingham, UK), G. Bazany-Barber, S. Fox, B. Fulton, J. McGrath, A. Smith, B. Wadsworth (University of York, UK), A. Boston, P. Nolan (University of Liverpool, UK), M. Junker (Laboratori Nazionali del Gran Sasso, Italy). 


\section{Introduction}

The idea of COBRA is to use a large array of CdZnTe semiconductor crystals to search for double beta decays[1]. CdZnTe contains 9 double beta isotopes, 5 of them being $\beta^{-} \beta^{-}$-emitters. Among them are ${ }^{130} \mathrm{Te}$ and ${ }^{116} \mathrm{Cd}$ as the most promising ones for the neutrino mass search and ${ }^{106} \mathrm{Cd}$ as one of only six known $\beta^{+} \beta^{+}$-emitters in nature. Advantages of the COBRA project are:

- Source is equal to detector. The isotopes of interest are intrinsic to the detector itself. This is always the most preferred approach.

- Semiconductor. Semiconductors are well known to have excellent energy resolution and can be produced very clean.

- Room temperature operation. For practical purposes, it is an enormous advantage to be able to operate the detectors without the need for cooling.

- Modular design. The modular design allows easy upgrades with only limited necessity for additional R\&D. In addition, it will allow significant background reduction, because high energy photons are likely to hit several detectors, while double beta decay is a single detector event.

- Two isotopes. The sensitivity in two isotopes will help to account partly for the NME uncertainties if $0 v \beta \beta$ decay is observed.

- The $\mathrm{Q}-\mathrm{value}$ of ${ }^{116} \mathrm{Cd}$ is beyond all relevant gamma-lines from natural decay chains, resulting in a significant background reduction.

- Pixelisation. The very exciting option of pixelisation would offer additional information in the form of tracking and vertex reconstruction. The device would act as a 'solid state TPC'.

- High sensitivity to 'Double- $\beta^{+}$' modes. Three different decay channels can be considered:

$$
\begin{gathered}
(Z, A) \rightarrow(Z-2, A)+2 e^{+}+\left(2 v_{e}\right) \\
e^{-}+(Z, A) \rightarrow(Z-2, A)+e^{+}+\left(2 v_{e}\right) \\
2 e^{-}+(Z, A) \rightarrow(Z-2, A)+\left(2 v_{e}\right)
\end{gathered}
$$

The last two cases involve electron capture (EC) which can only be seen within a 'source $=$ detector' approach. While it is assumed that $\beta^{+} / E C$ isotopes have longer half-lives than $\beta^{-} \beta^{-}$isotopes and are therefore less interesting for the determination of the neutrino mass, they are expected to yield important information for the disentanglement of the underlying mechanisms.

\section{Current status}

The data presented has been obtained with four $1 \mathrm{~cm}^{3} \mathrm{CdZnTe}$ detectors manufactured by eVPRODUCTS based on coplanar grid technology, i.e. reading out only the electron signal. Previous results based on a $0.5 \mathrm{~cm}^{3}$ commercial detector can be found in [2]. The measurements have been 
performed in the Gran Sasso Underground Laboratory (LNGS) in Italy providing a shielding of about 3500 mwe. The four bare detectors are mounted in a copper brick which is separated from all preamplifier electronics by about $25 \mathrm{~cm}$. The copper brick itself is part of a $(20 \mathrm{~cm})^{3}$ cube made out of electropolished copper and a further passive shielding of at least $15 \mathrm{~cm}$ of lead in all directions. The whole setup is located in a Faraday cage made from copper plates. The cage is surrounded by a neutron shield, consisting of $7 \mathrm{~cm}$ thick boron-loaded polyethylene plates and additional $20 \mathrm{~cm}$ of paraffin wax at the bottom. The full paraffin shielding was finished at a later stage.

The energy resolution and stability of the detectors is calibrated regularly with the help of ${ }^{137} \mathrm{Cs}$, ${ }^{60} \mathrm{Co}$ and ${ }^{228} \mathrm{Th}$ sources. The data were taken in three periods: First, a CAMAC-based DAQ system was used and a pertinax (FR2) crystal holder, which was replaced for the second period with a holder made out of delrin (POM). Additionally, the original Lemo cables for signal readout were replaced by a kapton (polyimide) based flexible PCB-cable. In a third step, the CAMAC DAQ was replaced by a custom-built VME-based DAQ system.

\section{Results}

Aside of double beta limits, a measurement of ${ }^{113} \mathrm{Cd}$ beta decay has been performed. The transition ${ }^{113} \mathrm{Cd} \rightarrow{ }^{113} \mathrm{In}$ is characterised as a $1 / 2^{+} \rightarrow 9 / 2^{+}$transition. Hence it is one of only a few 4 -fold forbidden non-unique decays ( $\Delta I=4$ and no change in parity). Their log ft-values are larger than 20 and correspondingly half-lives around $10^{15} \mathrm{yrs}$ and longer have to be measured. Only three isotopes of this type are known, ${ }^{50} \mathrm{~V},{ }^{113} \mathrm{Cd}$ and ${ }^{115} \mathrm{In}$. As can be seen in Fig. $1{ }^{113} \mathrm{Cd}$ spectra with an endpoint energy of about $320 \mathrm{keV}$ can be seen consistently in all four detectors. The final sample comprises of $0.86 \mathrm{~kg} \cdot \mathrm{d}$ and a half-life of

$$
\left.\left.T_{1 / 2}=(8.2 \pm 0.2 \text { (stat. })_{-1.0}^{+0.2} \text { (sys. }\right)\right) \cdot 10^{15} \quad \text { yrs }
$$

has been deduced. This half-life is in good agreement with the values quoted in [3, 4]. A comparison with the electron energy spectrum of the 'Table of Isotopes' Web-page ${ }^{1}$ has been performed. As can be seen in Fig. 1 the spectral shape is excluded by the data. The spectra are normalised to the total number of events in the range of 100-320 keV. For details see [5].

On the double beta side, a peak search for all $\beta \beta$-emitters has been done by using a maximum likelihood method. The total accumulated exposure corresponds to $3.82 \mathrm{~kg} \cdot \mathrm{d}$. The results are preliminary half-life limits of $1.18 \times 10^{19} \mathrm{yrs}\left({ }^{116} \mathrm{Cd}\right)$ and $5.67 \times 10^{19} \mathrm{yrs}\left({ }^{130} \mathrm{Te}\right)$ with $90 \% \mathrm{CL}$. New world best limits $\left(90 \% \mathrm{CL}\right.$ ) have been obtained for decays of ${ }^{64} \mathrm{Zn}$ and ${ }^{120} \mathrm{Te}$ : For the $0 \mathrm{v} \beta^{+} / E C$ and $0 v E C / E C$ ground state transitions of ${ }^{64} \mathrm{Zn}$ the limits are $5.07 \times 10^{18} \mathrm{yrs}$ and $9.52 \times 10^{16} \mathrm{yrs}$, respectively. For the $0 v \beta^{+} / E C$ and $0 v E C / E C$ ground state transitions of ${ }^{120} \mathrm{Te}$, the corresponding limits are $4.89 \times 10^{16} \mathrm{yrs}$ and $1.91 \times 10^{15} \mathrm{yrs}$.

\section{Outlook}

One significant method for background reduction due to the high granularity of the detectors should be coincidences. While double beta decay can be considered as a single detector event,

\footnotetext{
${ }^{1}$ see http://nucleardata.nuclear.lu.se/NuclearData/toi/
} 

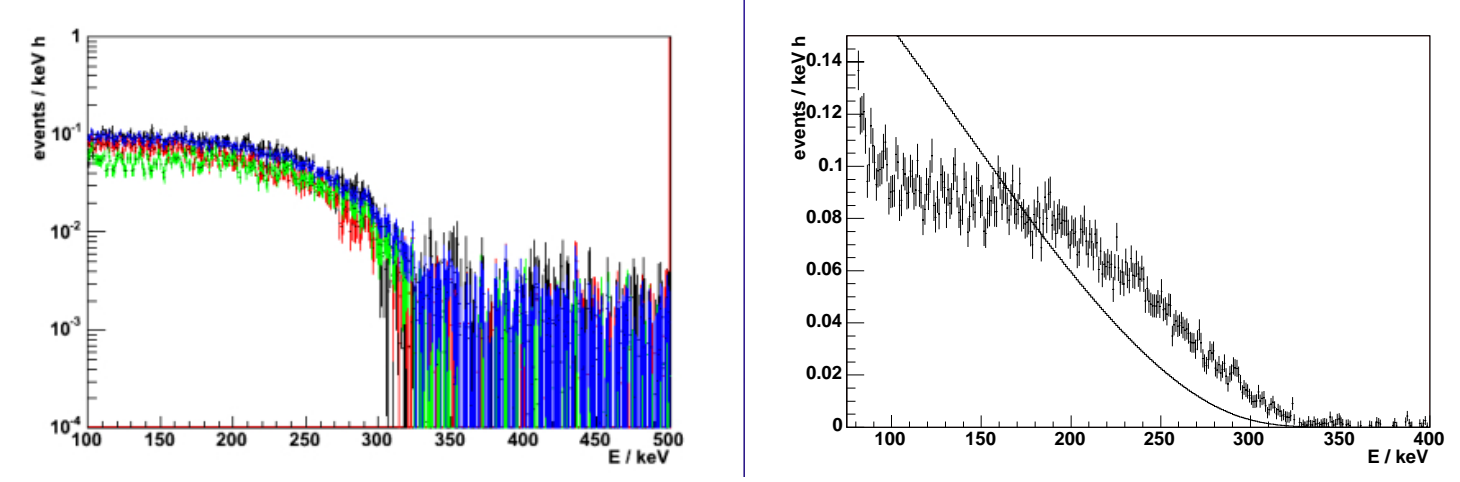

Figure 1: Left: Low energy spectra of all four CdZnTe detectors. As can be seen they all result in a consistent spectrum below $320 \mathrm{keV}$ due to ${ }^{113} \mathrm{Cd}$ decay. Right: Measured energy spectrum of the summed detectors (histogram) compared to the shape as expected from the 'Table of Isotopes' Web-page (line) indicating no good agreement. The spectra are normalised to the number of events between 100-320 keV.

high energy gamma rays will scatter around in the array. In the current set-up about $0.2 \%$ of the observed events are coincidences. However, as Monte Carlo simulations show this array is much too small to demonstrate the power of the coincidence technique. Hence, currently a $4 \times 4 \times 4$ array of $1 \mathrm{~cm}^{3} \mathrm{CdZnTe}$ detectors is in preparation which will have a total detector mass of about $0.5 \mathrm{~kg}$, an increase in detector mass by a factor 16 . Test measurements of all detectors are ongoing and installation of the array at LNGS will start by the end of 2005. An additional shielding component, in the form of liquid scintillator, is under investigation. This would actively veto backgrounds and also act as a signal enhancer for all excited state transitions and modes involving a $\beta^{+}$. Last but not least, work on pixelated detectors has started and first measurements have been performed.

The currently considered final design would consist of a $40 \times 40 \times 40$ array, enriched to $90 \%$ in ${ }^{116} \mathrm{Cd}$. This would result in a total mass of about $420 \mathrm{~kg}$ of CdZnTe.

\section{Summary}

COBRA is a new approach to double beta decay using CdZnTe semiconductor detectors. Since 2003, four CdZnTe detectors have been running in the Gran Sasso Underground Laboratory and first interesting results like a half-life measurement of ${ }^{113} \mathrm{Cd}$ have been obtained. An upgrade to 64 detectors with a total mass of about $0.5 \mathrm{~kg}$ will happen in the near future. Complementary work on pixelated detectors and active veto elements also acting as signal enhancers has started.

\subsection{Acknowledgments}

We thank the Laboratori Nazionali del Gran Sasso for their hospitality and support and V. Tretyak for providing us with the DECAY0 generator.

\section{References}

[1] K. Zuber, Phys. Lett. B 519, 1 (2001) 
[2] H. Kiel, D. Muenstermann and K. Zuber, Nucl. Phys. A 723, 499 (2003)

[3] A. Alessandrello et al., Nucl. Phys. B (Proc. Suppl.) 35, 394 (1994)

[4] F. Danevich et al., Phys. Atom. Nucl. 59, 1 (1994)

[5] C. Goessling et al., nucl-ex/0508016, acc. by Phys. Rev. C 